\title{
DNAJB2 Expression in Normal and Diseased Human and Mouse Skeletal Muscle
}

Kristl G. Claeys, ${ }^{\star \dagger}$ Magdalena Sozanska, ${ }^{\ddagger}$ Jean-Jacques Martin, ${ }^{\S}$ Emmanuelle Lacene, ${ }^{*}$ Ludivine Vignaud, ${ }^{\ddagger}$ Daniel Stockholm, ${ }^{\ddagger}$ Pascal Laforêt, ${ }^{\dagger}$ Bruno Eymard, ${ }^{\text {tTा }}$ Antoine Kichler, ${ }^{\neq \|}$Daniel Scherman, ${ }^{\ddagger \|}$ Thomas Voit, ${ }^{\dagger \star *}$ and David Israeli ${ }^{\ddagger}$

From the Unité de Morphologie Neuromusculaire," and the Centre de Référence Neuromusculaire Paris-Est ${ }^{\dagger}$ Institut de Myologie, Groupe Hospitalier Pitié-Salpêtrière, AP-HP, Paris, France; Généthon, ${ }^{\ddagger}$ Evry, France; the Laboratory of Ultrastructural Neuropathology, ${ }^{\S}$ Institute Born-Bunge, University of Antwerp, Antwerpen, Belgium; UPMC Paris 6," Paris, France; U640 INSERM - UMR 8151 CNRS," Faculté de Sciences

Pharmaceutiques et Biologiques, Paris, France; and UPMC UMR S974,** INSERM U974, CNRS UMR 7215, Institut de Myologie,

Paris, France

DNAJB2, a co-chaperone regulator of Hsp70 that is expressed principally in the nervous system, has been recently reported to be up-regulated in human skeletal muscle during its recovery from damage. Here we identified DNAJB2 expression in regenerating fibers in skeletal muscles of the dystrophic $\mathbf{m d x}$ mouse and patients with Duchenne muscular dystrophy. Surprisingly, in both dystrophic and control mice and patients, DNAJB2 was also expressed in non-regenerating fibers at the postsynaptic side of the neuromuscular junction. DNAJB2 functions as an adaptor molecule for the evacuation and degradation of proteins through the ubiquitin-proteasome system, and overexpression of DNAJB2 in models of the neurodegenerative disease spinobulbar muscular atrophy was shown to result in the reduction of protein inclusions. We therefore studied the possible relation of DNAJB2 expression to protein inclusion formation in skeletal muscle in biopsies of several muscle pathologies associated with protein aggregation and found in all of them a strong immunoreactivity with anti-DNAJB2 in aggregates and vacuoles. We conclude that DNAJB2 is expressed in mouse and human skeletal muscle at the neuromuscular junction of normal fibers, in the cytoplasm and membrane of regenerating fibers, and in protein aggregates and vacuoles in protein aggregate myopathies. Therefore, we propose a role for DNAJB2 in protein turnover processes in skeletal muscle. (Am J Patbol 2010, 176:2901-2910; DOI: 10.2353/ajpath.2010.090663)

The diverse members of the DNAJ/Heat shock protein 40 (Hsp40) family of co-chaperone proteins are characterized by a highly conserved 70-amino acids domain, called the "J domain." This domain interacts with Hsp70, which is a chaperone protein that plays a central role in the cellular stress response, and stimulates ATP hydrolysis. Thus, DnaJ proteins play an important role in the regulation of Hsp70 chaperone activity. Furthermore, several DnaJ proteins can regulate the activity of other chaperones, such as Hsp90, or have an independent chaperone activity (reviewed in Qiu ${ }^{1}$ ). Different DnaJ proteins are found in distinct subcellular compartments, including the cytoplasm, nucleus, mitochondria and endoplasmic reticulum.

Originally cloned from human brain tissue, DNAJB2 (designated also HSJ1) has been reported to be expressed principally in neurons. ${ }^{2}$ A recent study, however, reported the up-regulation of DNAJB2 transcript in human skeletal muscle during its recovery from exerciseinduced damage. ${ }^{3}$ Here, we studied DNAJB2 expression during muscle regeneration in the dystrophin-null $\mathrm{mdx}$ mouse, which is a well-known model to study muscle regeneration mechanisms (for a recent review see 4), and in patients with Duchenne muscular dystrophy (DMD). Indeed, we found expression of DNAJB2 in regenerating fibers, and surprisingly, we also identified expression in non-regenerating fibers, at the postsynaptic side of the neuromuscular junction (NMJ).

The DNAJB2 gene encodes two alternatively-spliced isoforms that differ in their $\mathrm{C}$-terminus. The variant 1 form

Supported by the Association Institut de Myologie (AIM), the Association Française contre les Myopathies (AFM), the Institut National de la Santé et de la Recherche Médicale (INSERM), and the Centre National de la Recherche Scientifique (CNRS).

Accepted for publication February 9, 2010

Supplemental material for this article can be found on http://ajp. amjpathol.org.

Address reprint requests to David Israeli, Ph.D., Généthon, 91002, Evry cedex, France. E-mail: israeli@genethon.fr. 
(V1 or HSJ1a) is expressed in both cytoplasm and nucleus, whereas the variant 2 form (V2 or HSJ1b) undergoes post-translational geranylgeranylation modification which mediates its attachment to the cytoplasmic side of the endoplasmic reticulum membrane. ${ }^{5,6}$ In addition, both alternatively spliced DNAJB2 isoforms possess ubiquitin interacting motifs that bind ubiquitinated proteins and target them to the proteasome for degradation. ${ }^{7}$ In accordance with a possible role of DNAJB2 in proteasomal degradation, it has recently been demonstrated that overexpressed DNAJB2 is highly effective in facilitating the removal of toxic protein aggregates in the nervous system. ${ }^{8}$ Protein aggregation also occurs within muscle fibers, notably in protein aggregate myopathies, of which the underlying molecular mechanisms are only partially understood. We therefore asked if the co-chaperone DNAJB2 was involved in the protein aggregation process in myopathies similar to its involvement in the central nervous system. We identified DNAJB2 immunoreactivity in protein aggregates in biopsies of several myopathies of different etiologies associated with protein aggregation, including sporadic and hereditary inclusion body myopathies and myofibrillar myopathies. The results presented here suggest a role of DNAJB2 at the NMJ in normal muscle and a possible involvement in the trafficking and degradation of abnormal muscle protein aggregates in myopathies.

\section{Materials and Methods}

\section{Mice}

We used the mdx4Cv model, which is an engineered mouse model carrying a missense mutation in exon 53 of the dystrophin gene (Jackson Laboratory, Bar Harbor, Maine), and C57BL/6 control mouse strains (Charles River Laboratories, Les Oncins, France). Skeletal and cardiac muscle, spinal cord, and brain tissues from C57BL/6 control mice were used for Western blot. For immunohistochemical and immunofluorescence techniques, skeletal muscle from both $\mathrm{mdx} 4 \mathrm{Cv}$ and C57BL/6 mice were used. All mice were handled in accordance with the European guidelines for use of experimental animals.

\section{Patients}

Tissues obtained from control individuals and patients used in the study are summarized in Table 1. Open skeletal muscle biopsies were performed, after informed consent, according to the Declaration of Helsinki.

\section{Western Blot Experiments}

Western blots were performed on tissues obtained from 5 weeks old C57BL/6 and mdx (CV4) mice strains, and from a 66-year-old autopsied patient. Tissues were mechanically homogenized in 10 volumes of $50 \mathrm{mmol} / \mathrm{L}$ Tris- $\mathrm{HCl} \mathrm{pH} \mathrm{7.5,} 5 \mathrm{mmol} / \mathrm{L}$ EDTA, $250 \mathrm{mmol} / \mathrm{L} \mathrm{NaCl}, 0.1 \%$ $\mathrm{NP} 40,5 \mathrm{mmol} / \mathrm{L}$ dithiothreitol, and $10 \mathrm{mmol} / \mathrm{L} \mathrm{NaF}$, con- taining protease inhibitors cocktail (1 697 498, Roche Biomedicals), and were kept on ice. The concentrations of the soluble protein (both mouse and cultured cellsderived extract) were determined by standard Bradford assay (Biorad) according to the instructions of the manufacturer. Protein extracts in Lameli loading buffer were heated during 5 minutes at $95^{\circ} \mathrm{C}$, chilled on ice, loaded on a precast $15 \%$ polyacrylamide gel (Biorad), and migrated about 90 minutes at $35 \mathrm{~mA}$. Migrated extracts were electro-transferred into a nitrocellulose membrane (Hybond ECL, Amersham Bioscience). The membrane was blocked overnight at $4^{\circ} \mathrm{C}$ with a blocking reagent (LI-COR, Cat. \#927-40000). Rabbit polyclonal anti-DNAJB2 (ProteinTech Group, 10838-1-AP, dilution 1:1500), mouse monoclonal anti- $\alpha$-tubulin (Sigma, T5168, dilution 1:5000), rabbit polyclonal antibody to glyceraldehyde-3-phosphate dehydrogenase (Santa Cruz FL335, 1:500), and anti-actin monoclonal (Sigma, clone AC-40, 1:5000) antibodies were added to the blocking mix and agitated during 1 hour at room temperature. After washing in PBS $0.1 \%$ Tween 20 , the membrane was hybridized with the secondary antibodies, anti-rabbit (IRDye 800, LI-COR) and anti-mouse (IRDye 680, LI-COR), diluted at 1:10,000, for 1 hour at room temperature. The membrane was washed several times in PBS $0.1 \%$ Tween 20 and scanned in the LI-COR Odyssey infrared two-color detection imager system (LI-COR Bioscience) according to manufacturer's instructions.

\section{Histological, Immunohistochemical, and Fluorescence Studies}

Specimens were processed using standard histological techniques. ${ }^{9}$ For conventional histological studies, $10-\mu \mathrm{m}$ thick frozen sections of unfixed material were stained with H\&E. For immunohistochemical studies, we used an immunoperoxidase automated procedure (Ventana, Illkirch, France), 8- $\mu \mathrm{m}$ thick cryosections in series with the corresponding H\&E sections, and primary antibodies directed against DNAJB2 (Proteintech Group, 10838-1-AP, polyclonal, rabbit, dilution 1:50), against another heat shock protein $\alpha$ B-crystallin (Novocastra, G2JF, polyclonal, rabbit, dilution 1:50), ubiquitin (Dako, polyclonal, rabbit, dilution 1:500), neonatal myosin (Novocastra, WB-MHCn, monoclonal, mouse, dilution 1:50), vimentin (Ventana, V9, monoclonal, mouse, dilution 1:1), NCAM or CD56 (Ventana, 123C3.D5, monoclonal, mouse, dilution 1:1), and utrophin (Novocastra, DRP3/20C5, mouse, monoclonal, dilution 1:20). In the immunofluorescence experiments, the same anti-DNAJB2 antibody (dilution 1:400) was used, either single or in double staining with $\alpha$-bungarotoxin (Molecular Probes, dilution 1:50) or with antibodies directed against neurofilament (neurofilamentheavy, Chemicon International, polyclonal, chicken, dilution 1:100). To rule out nonspecific immunoreactions, sections were incubated with secondary antibodies without the primary antibodies. 
Table 1. Control Individuals and Patients Included in the Immunohistochemical and Western Blot Studies

\begin{tabular}{|c|c|c|c|c|c|}
\hline & Diagnosis & Sex & Tissues & $\begin{array}{l}\text { Age at } \\
\text { biopsy (y) }\end{array}$ & IHC/WB \\
\hline & \multicolumn{5}{|c|}{ Controls } \\
\hline 1 & Normal & Female & Medial gastrocnemius muscle & 5 & $\mathrm{IH}$ \\
\hline 2 & Normal & Female & Paravertebral muscle & 12 & $\mathrm{IH}$ \\
\hline 3 & Normal & Female & Deltoid muscle & 35 & $\mathrm{IH}+\mathrm{WB}$ \\
\hline 4 & Normal & Female & Tibial anterior muscle & 29 & WB \\
\hline 5 & Normal & Male & Tibial anterior muscle & 60 & WB \\
\hline 6 & Normal & Male & Deltoid muscle & 37 & IH \\
\hline 7 & Normal & Female & Lateral vastus muscle & 44 & $\mathrm{IH}$ \\
\hline 8 & Normal & Female & Deltoid muscle & 56 & $\mathrm{IH}+\mathrm{WB}$ \\
\hline 9 & $\begin{array}{l}\text { Phosphorylase b kinase } \\
\text { deficiency* }\end{array}$ & Male & Deltoid muscle & 35 & $\mathrm{IH}$ \\
\hline 10 & Normal (autopsy; frozen) & Male & $\begin{array}{l}\text { Lateral vastus muscle, cardiac muscle (left } \\
\text { and right ventricle), lumbar spinal cord, } \\
\text { lumbar DRG, sural nerve }\end{array}$ & 66 & $\mathrm{IH}+\mathrm{WB}$ \\
\hline 11 & Normal (autopsy; frozen) & Male & $\begin{array}{l}\text { Lateral vastus muscle, cardiac muscle (left } \\
\text { and right ventricle), lumbar spinal cord, } \\
\text { lumbar DRG, sural nerve, sciatic nerve, } \\
\text { brain (frontal cortex, hippocampus) }\end{array}$ & 57 & WB \\
\hline 12 & Normal (autopsy; paraffin) & Male & Spinal cord & 35 & $\mathrm{IH}$ \\
\hline 13 & Normal (autopsy; paraffin) & Male & Brain (frontal cortex, hippocampus) & 63 & $\mathrm{IH}$ \\
\hline \multirow[t]{2}{*}{14} & Normal (autopsy; paraffin) & Male & Brain (frontal cortex, hippocampus) & 59 & $\mathrm{IH}$ \\
\hline & \multicolumn{5}{|c|}{ Patients } \\
\hline 15 & DMD & Male & Deltoid muscle & 2 & $\mathrm{IH}$ \\
\hline 16 & DMD & Male & Deltoid muscle & 4.5 & $\mathrm{IH}$ \\
\hline 17 & DMD & Male & Muscle unknown & 7 & $\mathrm{IH}$ \\
\hline 18 & DMD & Male & Muscle unknown & 10 & $\mathrm{IH}$ \\
\hline 19 & hIBM (IBMPFD-VCP) & Female & Deltoid muscle & 35 & $\mathrm{IH}$ \\
\hline 20 & hIBM (IBMPFD-VCP) & Male & Deltoid muscle & 61 & $\mathrm{IH}+\mathrm{WB}$ \\
\hline 21 & sIBM & Female & Tibial anterior muscle & 59 & $\mathrm{IH}$ \\
\hline 22 & slBM & Male & Radial muscle & 61 & $\mathrm{IH}$ \\
\hline 23 & slBM & Female & Lateral vastus muscle & 71 & $\mathrm{IH}$ \\
\hline 24 & sIBM & Female & Medial gastrocnemius muscle & 71 & $\mathrm{IH}$ \\
\hline 25 & sIBM & Female & Deltoid muscle & 75 & $\mathrm{IH}+\mathrm{WB}$ \\
\hline 26 & SIBM & Female & Tibial anterior muscle & 71 & $\mathrm{IH}$ \\
\hline 27 & sIBM & Female & Tibial anterior muscle & 72 & $\mathrm{IH}+\mathrm{WB}$ \\
\hline 28 & MFM (desmin) & Male & Sural triceps muscle & 38 & $\mathrm{IH}$ \\
\hline 29 & MFM (desmin) & Male & Deltoid muscle & 52 & $\mathrm{IH}$ \\
\hline 30 & MFM ( $\alpha$ B-crystallin) & Female & Deltoid muscle & 36 & $\mathrm{IH}$ \\
\hline 31 & MFM (myotilin) & Male & Tibial anterior muscle & 62 & $\mathrm{IH}$ \\
\hline 32 & MFM (myotilin) & Male & Tibial anterior muscle & 71 & WB \\
\hline 33 & MFM (ZASP) & Male & Lateral vastus muscle & 54 & $\mathrm{IH}$ \\
\hline
\end{tabular}

y, years; IH, immunohistological study; WB, Western blot study; DRG, dorsal root ganglion; DMD, Duchenne muscular dystrophy; slBM, sporadic inclusion body myositis; hIBM, hereditary inclusion body myopathy; IBMPFD-VCP, inclusion body myopathy with Paget's disease of bone and frontotemporal dementia caused by a mutation in the gene encoding valosin containing protein; MFM, myofibrillar myopathy; ZASP, Z-band alternatively spliced PDZ-motif containing protein; ${ }^{*}$, no morphological abnormalities and included in the study because of the large number of neuromuscular junctions in the muscle biopsy.

\section{Results}

\section{Western Blot Studies: DNAJB2 Expression in C57BL/6 and mdx4Cv Mice and in Human Controls}

Western blot analysis was used to assay expression of DNAJB2 in skeletal muscle derived from wild-type C57BL/6 mouse, compared with expression in regenerating muscle derived from 5-week-old $\mathrm{mdx} 4 \mathrm{Cv}$ mouse. DNAJB2 was expressed in all muscle samples, V2 being the predominant isoform. No differences in the muscle expression level of DNAJB2 were found between the two strains (Figure 1A). Western blot analysis was also used to compare the relative expression level of DNAJB2 in different tissues in mouse and human (Figure 1, B and C).
In agreement to previous publications, ${ }^{2,5}$ we found that in the mouse the highest expression level (normalized to protein quantity) was in the brain, followed by the spinal cord, and cardiac and skeletal muscles (Figure 1B). In most studied tissues, expression of the larger alternatively spliced isoform V2 (38 kDa) largely exceeded the expression of the $\mathrm{V} 1$ isoform ( $32 \mathrm{kDa}$ ), which was below our detection level in some tissues and experiments. As in the mouse, also in human the highest expression level of DNAJB2 was found in the frontal cortex, followed by the other neural tissues and largely exceeded the level in cardiac and skeletal muscles (Figure 1C). Interestingly, in human skeletal muscle, in contrast to mouse skeletal muscle and to all other tissues, the $\mathrm{V} 1$ isoform expressed equally and sometimes even stronger than the alternatively spliced V2 isoform. 


\section{A}

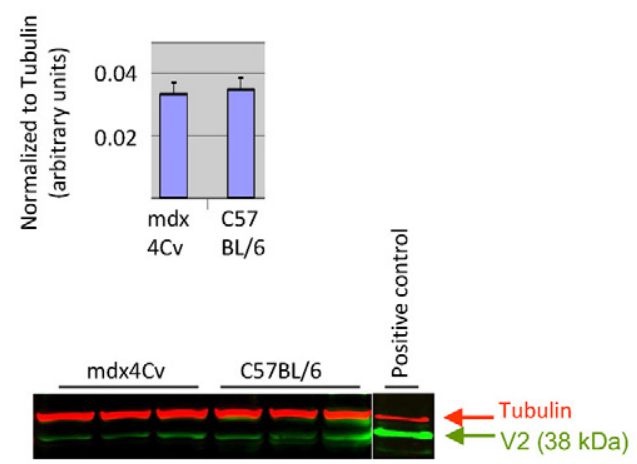

C
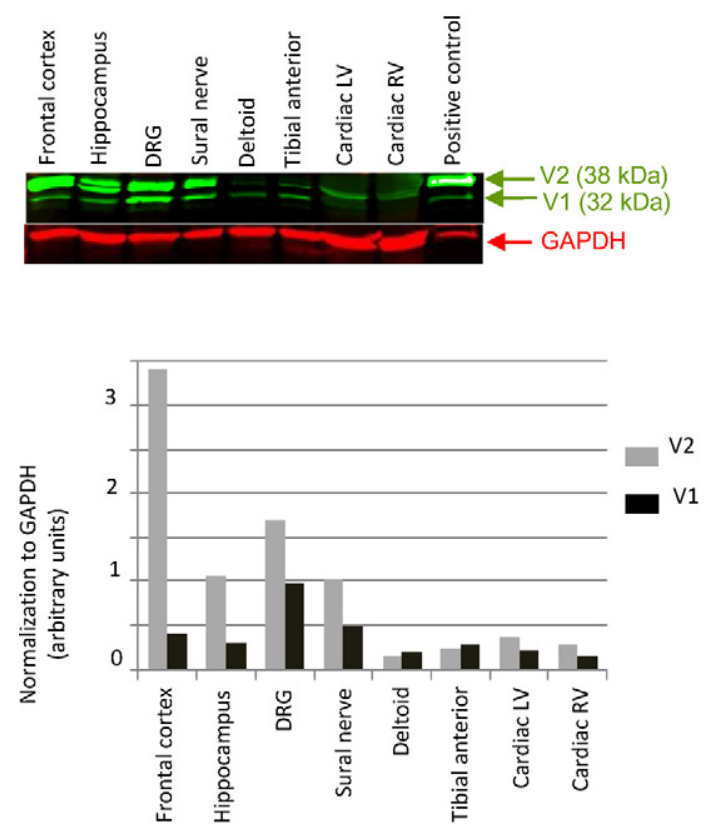

B
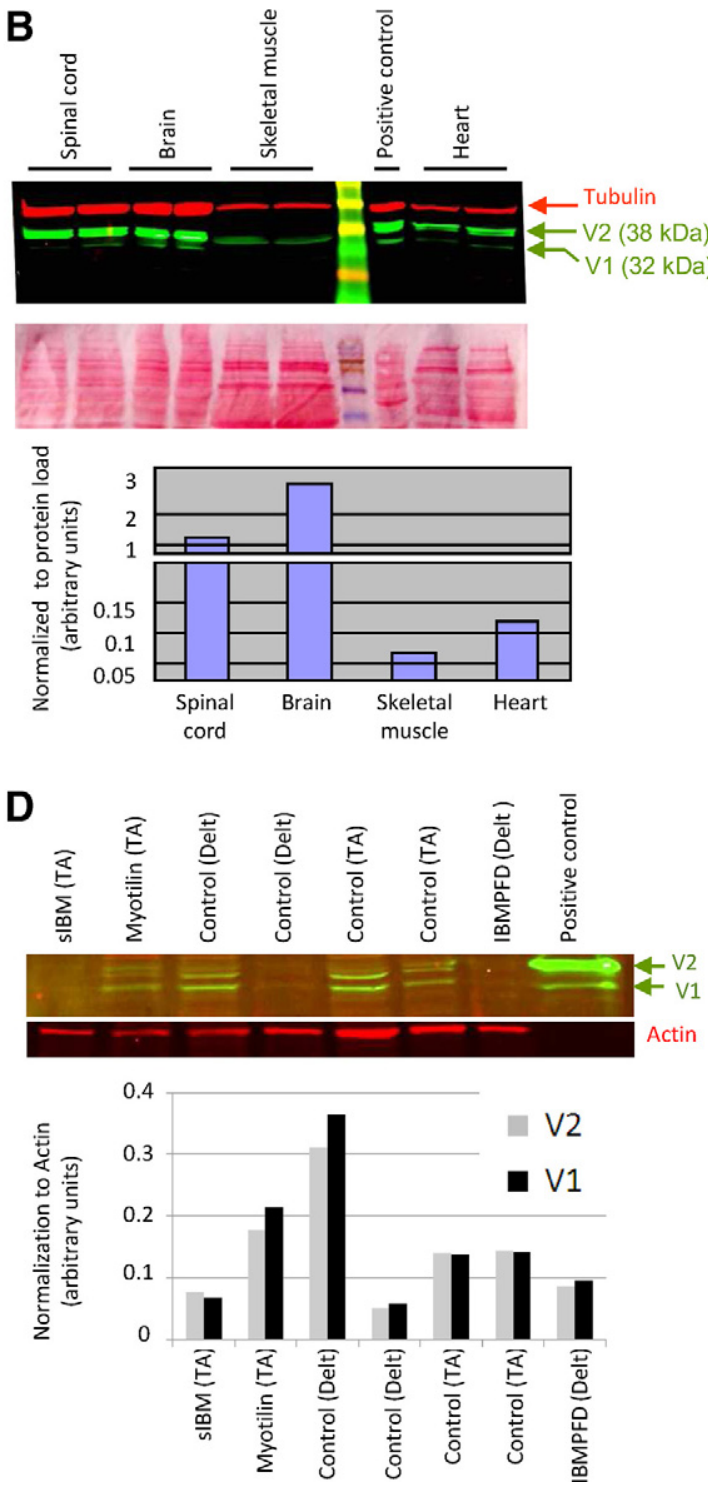

Figure 1. Western blots of DNAJB2 expression in mice and control patients. A: Western blot and expression level of DNAJB2 in skeletal muscle of dystrophic mdx $4 \mathrm{Cv}$ and control $\mathrm{C} 57 \mathrm{BL} / 6$ mice show no significant differences. Expression level was normalized to $\alpha$-tubulin. B-C: Western blot and relative expression level of DNAJB2, are shown for the different tissues analyzed in mouse (B) and human (C). Positive control is an extract of C2 myoblast cotransfected by DNAJB2 V1 and V2 expression plasmids. D: Western blot and expression level of DNAJB2 in skeletal muscle of patients with sIBM, IBMPFD, and myofibrillar myopathy (myotilin mutation) and of controls. The green arrows indicate the two alternatively spliced isoforms, V1 and V2, of the DNAJB2 protein. DRG, dorsal root ganglion; LV, left ventricle; RV, right ventricle; TA, tibial anterior; Delt, deltoid muscle.

\section{Histological Analyses of DNAJB2 Expression in C57BL/6 Control Mice}

A very strong immunoreactivity was revealed at the neuromuscular junction in normal control mice using the antibody directed against DNAJB2 (Figure 2, A, D and G). Labeling with $\alpha$-bungarotoxin for the neuromuscular junction (Figure 2, B, E, and $\mathrm{H}$ ), revealed complete colocalization, thus confirming the postsynaptic expression of DNAJB2 (Figure 2, C, F and I). In normal myofibers a very weak, non-specific diffuse sarcoplasmic staining was revealed using anti-DNAJB2 (Figure 2, A and D). In contrast, a specific DNAJB2 immunoreactivity was revealed in the sarcolemma of some muscle fibers tips in a close vicinity to their perimysial attachment, (See Supplemental Figure S1, A and C at http://ajp. amjpathol.org), as well as in the sarcoplasm and sarcolemma of small intrafusal (spindle) fibers (See Supplemental Figure S1, D-F at http://ajp.amjpathol.org). The isolated sciatic peripheral nerve showed immunoreactivity for DNAJB2, which was colocalized with antineurofilament reactivity, thus corresponding to an axonal localization of DNAJB2 (Figure 2, J-O). In addition, immunofluorescence reactivity for anti-DNAJB2 in the axons of small psoas nerve branches situated on transverse sections through the psoas muscle, was present but weaker than that in isolated peripheral nerve and at the neuromuscular junction (Figure 2, P-U). More detailed analysis confirmed the presence of DNAJB2 in the axons of psoas nerve branches in muscle tissue (Figure 2, S-U, and Supplemental Figures S2 and S3 at http://ajp.amjpathol.org). 

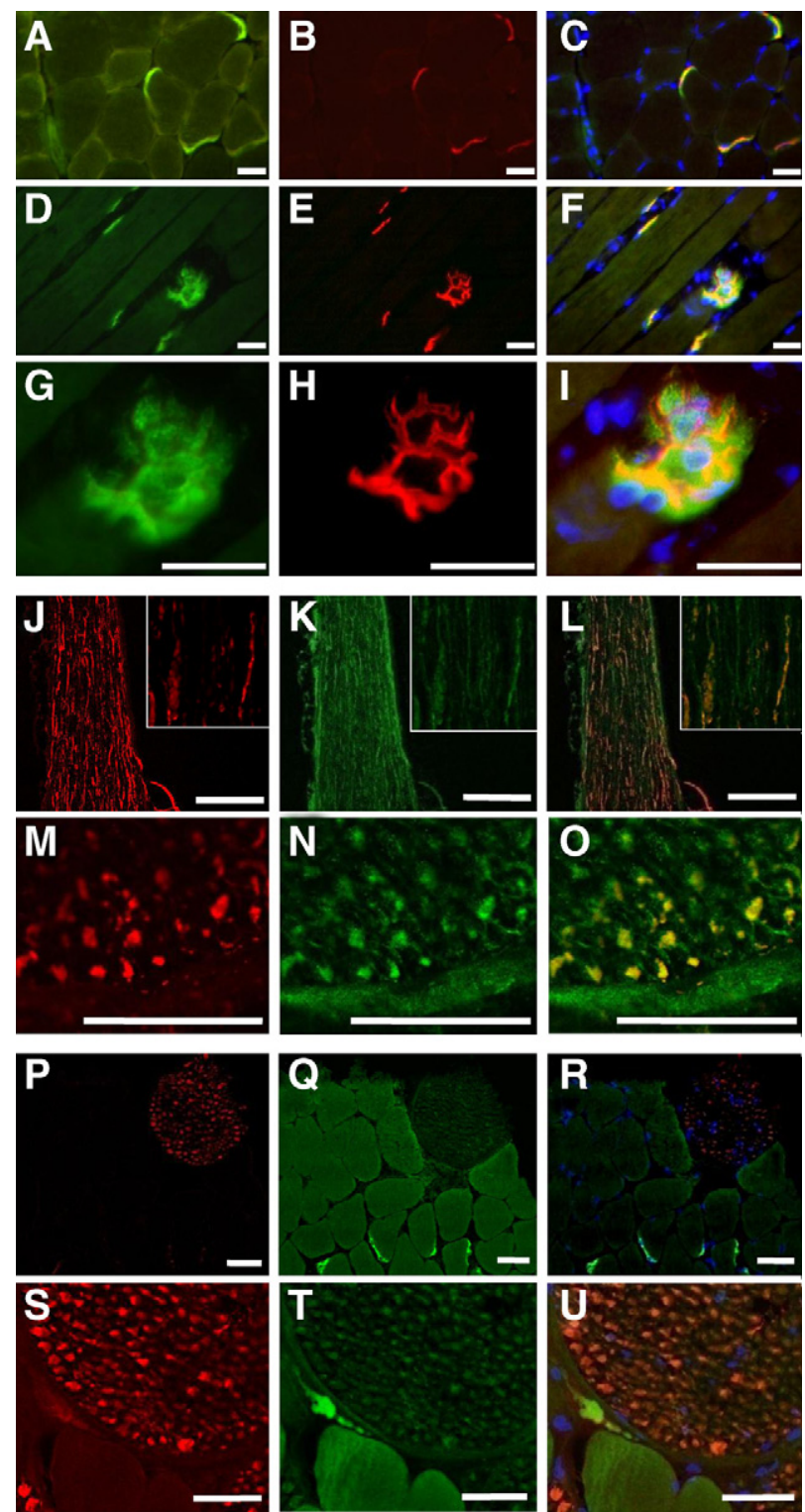

Figure 2. DNAJB2 immunoreactivity in normal control C57BL/ 6 mice. A-I Serial transverse $(\mathbf{A}-\mathbf{C})$ and longitudinal $(\mathbf{D}-\mathbf{I})$ sections of the tibial anterior muscle in wild-type mice. DNAJB2 is expressed at the NMJ, shown by double staining with DNAJB2 (green; A, D, G), $\alpha$-bungarotoxin (red; $\mathbf{B}, \mathbf{E}, \mathbf{H}$ ), and their co-staining (yellow; $\mathbf{C}, \mathbf{F}, \mathbf{I}$ ). The branched structure of the NMJ postsynaptic membrane observed in (D-F) is shown at a larger magnification in (G-I). Nuclei are stained with 4,6-diamidino-2-phenylindole (DAPI; blue; C, $\mathbf{F}, \mathbf{I})$. Scale bars $=50 \mu \mathrm{m}$. J-O: Isolated sciatic nerve is shown on longitudinal (J-L) and transverse (M-O) sections, with anti-neurofilament (red; $\mathbf{J}, \mathbf{M}$ ), DNAJB2 (green; K, N), and double staining (orange-yellow; $\mathbf{L}, \mathbf{O}$ ). The inset represents a larger magnification of a part of the nerve shown in $\mathbf{J}-\mathbf{L}$. Bars indicate a magnification of $200 \mu \mathrm{m}(\mathbf{J}-\mathbf{L})$ and $25 \mu \mathrm{m}(\mathbf{M}-\mathbf{O})$. P-U: A small nerve branch on a transverse section through the psoas muscle reveals anti-DNAJB2 immunofluorescence $(\mathbf{Q}, \mathbf{T})$ that is weaker than in the NMJ Axonal colocalization (orange; $\mathbf{R}, \mathbf{U}$ ) of anti-DNAJB2 (green; $\mathbf{Q}, \mathbf{T}$ ) with anti-neurofilament (red; $\mathbf{P}, \mathbf{S}$ ) is found. Scale bars $=50 \mu \mathrm{m}$.

\section{Histological Analyses of DNAJB2 Expression in Dystrophic mdx4Cv-Mice}

A strong immunoreactivity was revealed in the sarcoplasm and at the sarcolemma of small muscle fibers with internalized large vesicular nuclei and bluish appearance at H\&E stains, corresponding to regenerating fibers (Fig- ure 3, A-F). Similar to normal muscle, neuromuscular junctions in $\mathrm{mdx}$-mice showed a strong expression of DNAJB2 (Figure 3, C, F, G and H). Colocalization with $\alpha$-bungarotoxin confirmed the postsynaptic expression of DNAJB2 (Figure 3, I-L). Double staining with the antibody directed against DNAJB2 and $\alpha$-bungarotoxin revealed co-expression of these two proteins in regenerating fibers, which is most pronounced at the sarcolemma (Figure $3, M-P)$.

\section{Histological Analyses of DNAJB2 Expression in Human Controls}

At the neuromuscular junction a very strong anti-DNAJB2 reactivity was observed (Figure $4, A-C$ ), which was absent at the myotendinous junction (Figure 4, G-H). Consistent with the mice results, the sarcoplasm in normal mature skeletal muscle fibers stained negative with antiDNAJB2, however, sometimes a very weak, diffuse, nonspecific immunoreaction can be found. In contrast, (but consistently with the mouse study) a specific weak diffuse immunoreactivity with anti-DNAJB2 was revealed at the sarcoplasm of the intrafusal muscle fibers (Figure 4 , $\mathrm{M}-\mathrm{N}$ ). The axons of perimysial nerves (Figure 4, K-L, $\mathrm{O}-\mathrm{R}$ ) and of the isolated sural nerve (Figure 4, D-F), showed immunoreactivity with anti-DNAJB2, whereas the myelin sheaths remained unstained. Furthermore, in the axons/dendrites and cell bodies of sensory neurons in the dorsal root ganglion (data not shown), and in the nerve axons and soma of motor neurons in the ventral horn of the spinal cord (Figure 4, I-J), strong immunoreactivity for anti-DNAJB2 was revealed. In fact, DNAJB2 was strongly expressed in both white and gray matter of the spinal cord (See Supplemental Figure S4, A-C at http://ajp.amjpathol.org). Intramedullar DNAJB2 staining was stronger compared with that in the nerve roots (See Supplemental Figure S4D at $h$ ttp://ajp.amipathol.org). In the latter, DNAJB2 immunoreactivity was particularly observed in the axons (See Supplemental Figure 4, E-F at http://ajp.amjpathol.org).

\section{Histological Analyses of DNAJB2 Expression in $D M D$}

The biopsies of the four DMD patients revealed a strong, diffuse immunoreactivity with anti-DNAJB2 in regenerating fibers (Figure 5, A-B). These regenerating muscle fibers also react with neonatal myosin and vimentin antibodies (Figure 5, C-H). In addition, a few fibers without obvious abnormalities by H\&E stains showed antiDNAJB2 immunoreactivity in their sarcoplasm. In contrast to normal muscle fibers, the sarcolemma of a large number of fibers in DMD patients was slightly and irregularly stained with the DNAJB2 antibody.

\section{Histological Analyses of DNAJB2 Expression in Human Protein Aggregate Myopathies}

Immunostaining with anti-DNAJB2 in the biopsies of the two patients with hereditary inclusion body myopathy, Paget's 
A
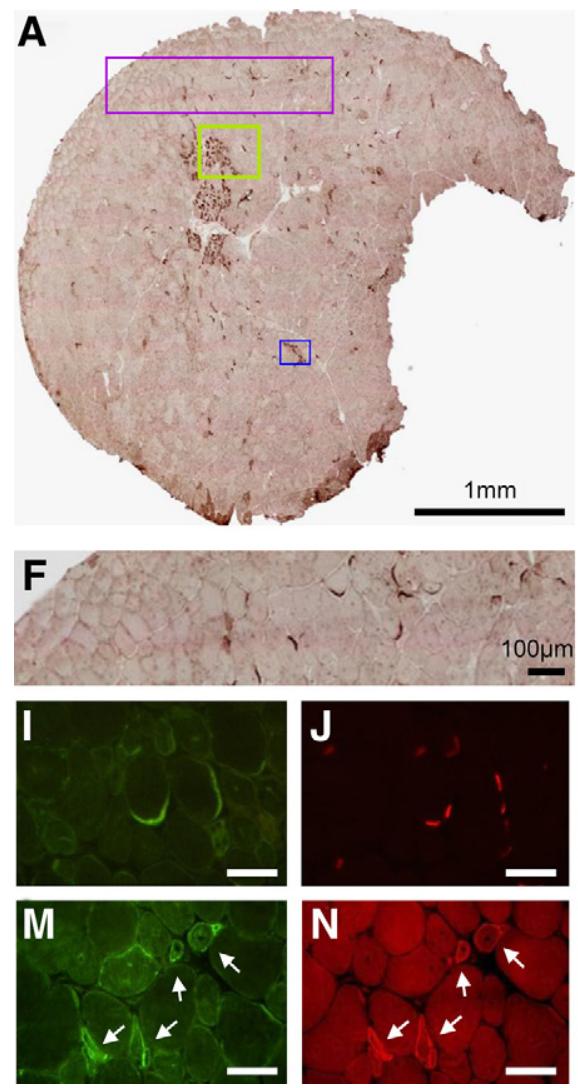
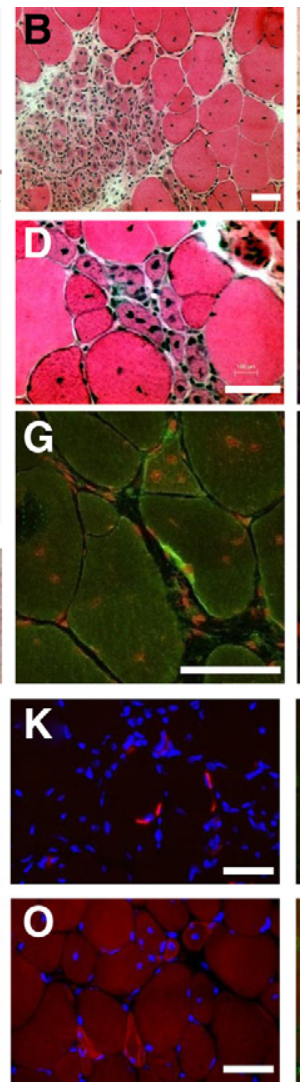
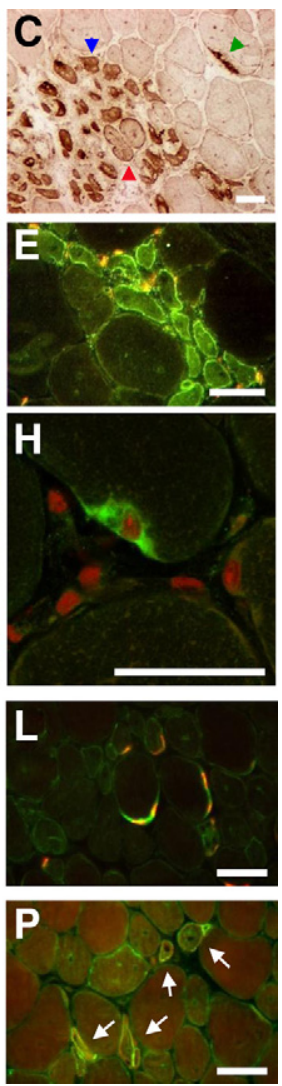

Figure 3. Expression of DNAJB2 in dystrophic muscle of the mdx $4 \mathrm{Cv}$ mice. A: DNAJB2 staining of transverse section of tibial anterior muscle in mdx mouse. The green box in (A) is seen at a higher magnification in (B-C) by H\&E (B) and anti-DNAJB2 immunohistochemical stains (C). Similarly, the blue box in (A) is shown at a higher magnification in (D-E) by H\&E stain (D) and DNAJB2 immunofluorescence (E). Notice that DNAJB2 is diffusely expressed in the sarcoplasm and at the sarcolemma of small regenerating fibers (blue arrowhead), whereas in slightly larger regenerating fibers its expression is mainly observed at the sarcolemma (red arrowhead). In $\mathrm{mdx}$ mouse, strong expression of DNAJB2 is revealed at the neuromuscular junction (green arrowhead in C). DNAJB2 in NMJs is seen further in the purple box (A) and at a higher magnification (F), and by confocal imaging $(\mathbf{G}-\mathbf{H}$; DNAJB2 in green. Nucle are shown in red (propidium iodide)). I-L: Double immunostaining at NMJs is shown: anti-DNAJB2 in green (I), $\alpha$-bungarotoxin in red ( $\mathbf{J}), \alpha$-bungarotoxin merged with DAPI (blue, $\mathbf{K}$ ) and merged with anti-DNAJB2 (yellow, L). M-P: Co-expression of DNAJB2 and $\alpha$-bungarotoxin is seen (white arrows) at the sarcolemma of regenerating fibers (DNAJB2, green, $\mathbf{M} ; \alpha$-bungarotoxin, red, $\mathbf{N}$; $\alpha$-bungarotoxin merged with DAPI, blue, $\mathbf{O}$ $\alpha$-bungarotoxin merged with anti-DNAJB2, yellow $(\mathbf{P})$. Scale bars $=50 \mu \mathrm{m}$, except $(\mathbf{H})$ in which the bar corresponds to $25 \mu \mathrm{m}$. disease and frontotemporal dementia caused by a mutation in VCP (IBMPFD), revealed a single or multiple areas with strong irregular immunoreactivity within the muscle fibers, underneath the sarcolemma and/or in the sarcoplasm (Figure $5,1-J)$. These areas corresponded to the aggregations observed at H\&E stains. Immunoreaction was also seen at the border of the vacuoles in the muscle fibers, as well as inside the vacuoles showing a punctiform or granular staining pattern. The accumulations and vacuoles also showed immunoreactivity for the antibody directed against $\alpha$ B-crystallin (data not shown), which is another heat shock protein. Some of the severely affected muscle fibers additionally presented an immunoreactivity of their sarcoplasm. In contrast to normal muscle, the sarcolemma in IBMPFD was slightly stained, in a discontinuous manner, in most affected myofibers but also in seemingly unaffected fibers.

In the seven patients with sporadic inclusion body myositis, immunoreactivity for anti-DNAJB2 was revealed in the fiber areas containing aggregations at H\&E stains, around vacuoles, and at the sarcolemma of affected and seemingly normal muscle fibers (Figure 5, K-P). Immunoreactivity with an antibody directed against $\alpha \mathrm{B}$-crystallin, which was tested in one slBM patient, was also revealed in the aggregates and vacuoles in this patient's biopsy (data not shown).

The findings in the five patients with myofibrillar myopathy caused by mutations in desmin, $\alpha \mathrm{B}$-crystallin, myotilin, or ZASP were largely similar to those in IBMPFD. In the patient with ZASPopathy, serial sections revealed immunoreactivity in the aggregations observed with $\mathrm{H} \& \mathrm{E}$ stains with the antibodies directed against DNAJB2, $\alpha \mathrm{B}$ crystallin and ubiquitin (Figure 5, Q-T).

\section{DNAJB2 Expression Is Not Up-Regulated in Protein Aggregate Myopathies (sIBM, IBMPFD, Myofibrillar Myopathy)}

A semiquantitative Western blot analysis has been used to study the relative level of DNAJB2 expression in muscle of patients with sIBM, IBMPFD, and myofibrillar myopathy, compared with controls. Although in some of the muscle biopsies, the DNAJB2 signal was at the limit of visual detection, specific DNAJB2 fluorescence signal could be measured in all samples. After normalization to the expression of actin, we have identified a variation of up to sixfold in expression level of DNAJB2 between the different muscle biopsies (Figure 1D), which however did not correlate with the presence of protein aggregates.

\section{Summary of Main Histological Findings in Human and Mice}

DNAJB2 in mouse and human skeletal muscle is localized at the postsynaptic neuromuscular junction in normal mature myofibers, and at the sarcoplasm and sarcolemma in regenerating fibers. DNAJB2 accumulation is 

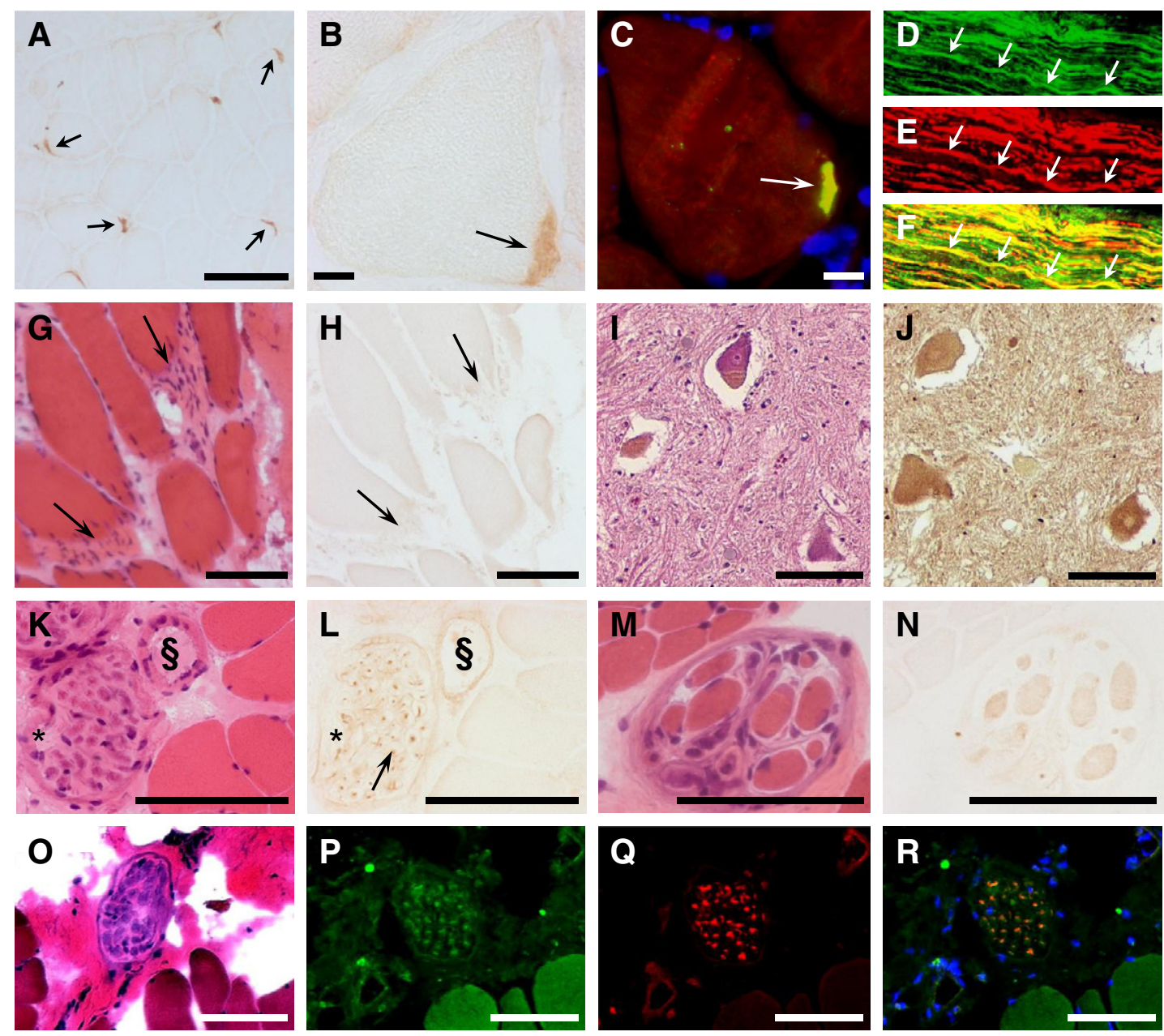

Figure 4. DNAJB2 immunoreactivity in normal human tissues. A-B: NMJs (arrows) in control muscle (control 9; see Table 1) show strong immunoreactivity for the DNAJB2 antibody, revealed by immunohistochemical (A-B) and immunofluorescence $(\mathbf{C})$ techniques. Co-localization of DNAJB2 with $\alpha$-bungarotoxin at the neuromuscular junction is shown in yellow (C arrow). D-F: A longitudinal section through a normal sural nerve (control 10) reveals axonal colocalization (yellow) (F) of DNAJB2 (D) and neurofilament (E). One axon is indicated by arrows. G-H: Myotendinous junctions (arrows) in a normal control biopsy (control 2) reveal no immunoreactivity for anti-DNAJB2, as indicated on serial transverse sections at H\&E stains (G) and anti-DNAJB2 immunoreactivity (H). I-J: Transverse sections through the ventral horn of the spinal cord (control 12) at H\&E (I) and DNAJB2 (J) stains, reveal anti-DNAJB2 immunoreactivity in the soma of motoneurons and in axons. K-L: Normal control skeletal muscle (control 6) shows muscle fibers with a very weak sarcoplasmic immunoreactivity using the anti-DNAJB2 antibody, at serial transverse sections with H\&E (K) and DNAJB2 (L) stains. Reactivity for anti-DNAJB2 in the axons (arrow) of a transversally cut perimysial nerve (asterisk) and in the wall of a perimysial blood vessel $(\boldsymbol{\$})$ are shown. $\mathbf{M}-\mathbf{N}$ : Intrafusal fibers of a muscle spindle are slightly stained with anti-DNAJB2, shown on transverse serial sections through normal control muscle (control 1) by H\&E (M) and DNAJB2 (N) stains. O-R: Serial transverse muscle sections (control 6) show a perimysial nerve at H\&E stains $(\mathbf{O})$, and fluorescence staining with anti-DNAJB2 (P), anti-neurofilament (Q), and their co-staining (R). The myonuclei are stained in blue (DAPI). Scale bars $=100 \mu \mathrm{m}$, except $(\mathbf{B}, \mathbf{C})$ in which the bar corresponds to $10 \mu \mathrm{m}$.

found in sarcoplasmic protein aggregates in diverse human protein aggregation myopathies.

\section{Discussion}

DNAJB2 has been reported to be enriched in neurons and in the nervous system. Indeed, most published studies about DNAJB2 have been focused on its expression and function in neuronal tissues. In agreement, our Western blot analysis of the relative level of DNAJB2 in skeletal and cardiac muscles, and in several neuronal tissues, confirmed a much higher expression level in all studied neuronal tissues, as compared with muscle samples.

A recent paper ${ }^{3}$ reported the activation of the DNAJB2 transcript in biopsies taken from muscle of human volunteers subjected to eccentric effort. The significance of these data however was not clear, since this transcriptomic study did not provide details about mRNA or protein expression levels of muscle DNAJB2 (only its mRNA activation), nor on the site of expression in muscle. Skeletal muscle is a complex structure that, in addition to pure myogenic contractile material, is also composed of blood vessels, hematopoietic cells, connective and neuronal structures, each of which (in particular the neuronal structures) could potentially be at the origin of the musclederived DNAJB2 transcript.

To test the activation of DNAJB2 in regenerating muscle we analyzed muscle of 5-week- old mdx mice, which is a very active muscle regeneration period in this model. The result of this experiment did not support an upregulation of DNAJB2 in regenerating muscles. However, in our histological studies, we have identified a shift in the 

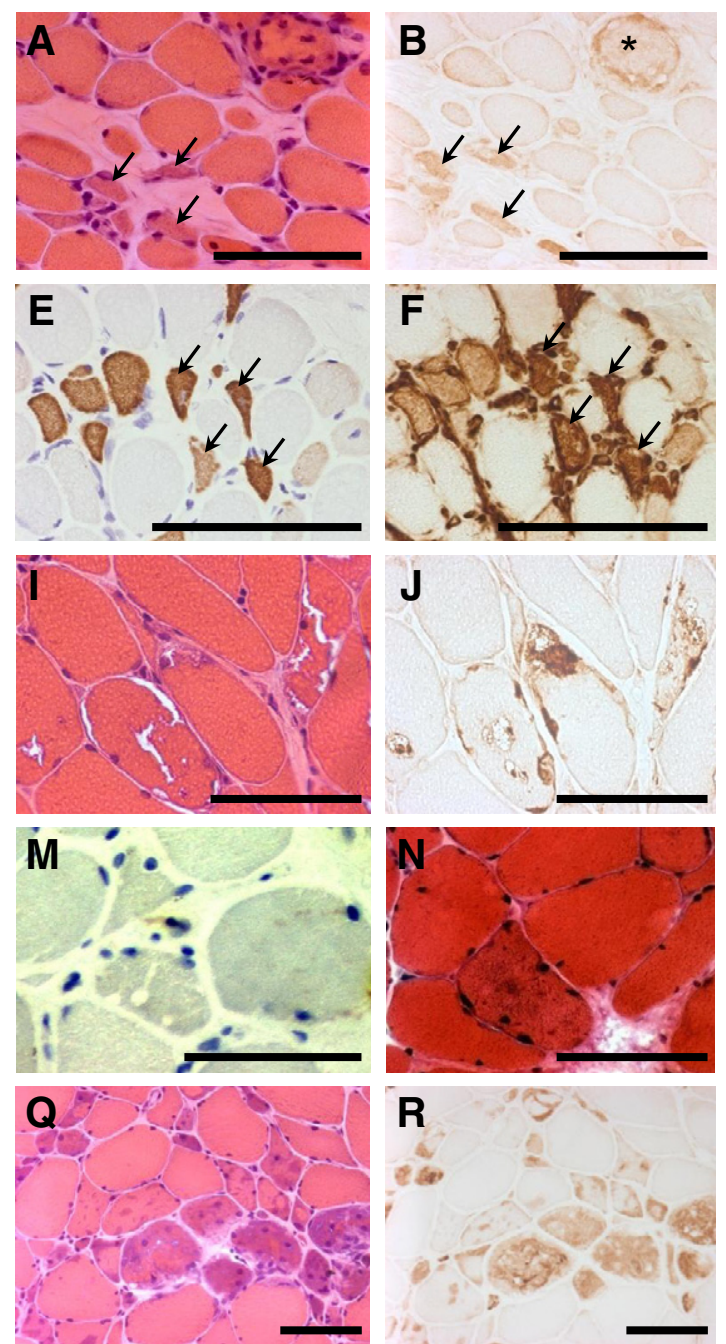
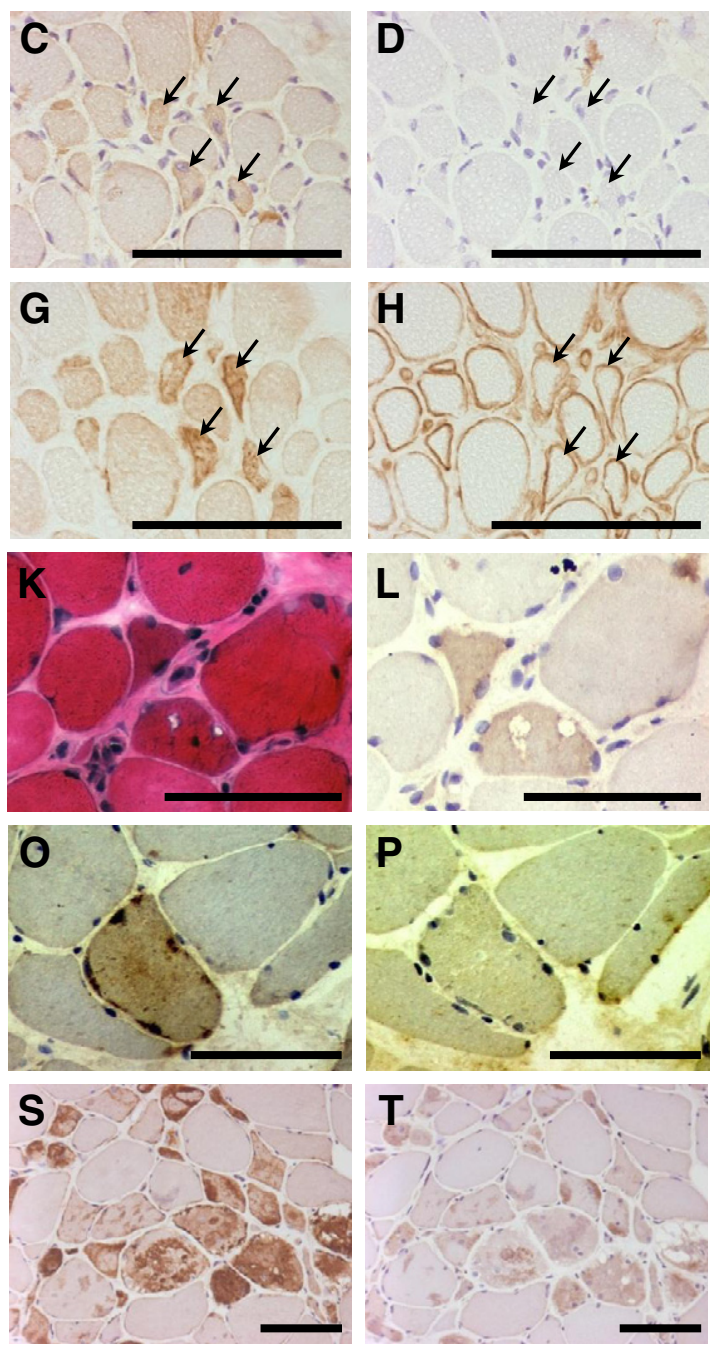

Figure 5. DNAJB2 immunoreactivity in Duchenne muscular dystrophy and in protein aggregate myopathies. A-B: The biopsy of a patient with DMD reveals immunoreactivity for anti-DNAJB2 in regenerating fibers (arrows) and in a necrotic fiber (asterisk). Serial transverse sections with H\&E (A) and DNAJB2 (B) stains are shown. C-H: Serial sections indicating regenerating fibers (examples indicated with arrows) at stains with antibodies against DNAJB2 (C), without first antibody (D), neonatal myosin (E), vimentin (F), NCAM (G), and utrophin (H). I-J: In a patient with IBMPFD (patient 20) immunoreactivity with anti-DNAJB2 is revealed in the aggregations, in and around the vacuoles, and at the sarcolemma of muscle fibers, as indicated on serial transverse sections with H\&E (I) and DNAJB2 (J) stains. K-P: In sIBM patients DNAJB2 immunoreactivity is revealed in some muscle fibers and around vacuoles, as shown on serial sections with H\&E (K), DNAJB2 stains (L), and without primary antibody $(\mathbf{M})$ in patient 27 , and in aggregations as shown on serial sections with H\&E (N), DNAJB2 stains $(\mathbf{O})$ and without primary antibody $(\mathbf{P})$ in patient 25 . Q-T: Serial transverse sections in a patient with myofibrillar myopathy caused by a mutation in ZASP (patient 33) reveal immunoreactivity in the aggregates observed with H\&E stain $(\mathbf{Q})$ for the antibodies against DNAJB2 (R), $\alpha \mathrm{B}$-crystallin $(\mathbf{S})$, and ubiquitin $(\mathbf{T})$. Scale bars $=100 \mu \mathrm{m}$.

site of expression of DNAJB2 in myofibers, in correlation with the fiber maturation stage: initially in the cytoplasm, later at the entire sarcolemma and finally locally at the NMJ. This pattern is not unique for DNAJB2 and has been found for many other junctional proteins, including the archetypal acetylcholine receptor (Reviewed in 10). It is assumed that in young myofibers the junctional protein transcripts are synthesized nonselectively by the myofiber nuclei and that only after the maturation of the NMJ, exclusively the junctional-specific nucleus continues to synthesize these transcripts.

The absence of DNAJB2 expression activation in our study, compared with the reported activation of DNAJB2 in regenerating human muscle, could result merely from interspecies differences. Alternatively, differences might result from the two different muscle regeneration paradigms and detected events (transcript versus protein) used in the two studies. In particular, regeneration was temporally synchronized in the human paradigm, ${ }^{3}$ which is not the case for regenerating muscle in the $\mathrm{mdx}$ mouse. All together, it seems as if the expression pattern of DNAJB2 in regenerating fibers is similar to that reported for some other NMJ postsynaptic proteins and that DNAJB2 up-regulation during regeneration will depend on the studied system.

To further explore DNAJB2 expression in skeletal muscles we have additionally studied human biopsies. Initially, the histological study of muscle biopsies derived from the DMD patients validated and generalized the pattern observed at the mdx mouse model, namely expression of DNAJB2 in the sarcoplasm and sarcolemma of regenerating fibers. However in the neuronal system, the biological role of DNAJB2 has been linked to the protein degradation system and aggregation experimen- 
tal paradigms, rather than to regeneration. DNAJB2 has been reported to serve as a shuttle factor for the delivery of ubiquitinated proteins to degradation by the ubiquitin proteasome system (UPS) and to participate in the endoplasmic reticulum-associated degradation (ERAD) system in the removal of toxic proteins from the endoplasmic reticulum. ${ }^{7}$ Specifically, an involvement of DNAJB2 in the prevention of protein aggregation in neuronal model systems has been shown in vitro in a cellular model of rhodopsin processing, ${ }^{5}$ Huntington's disease, ${ }^{7}$ and in vitro and in vivo in models of protein aggregation in the neurodegenerative pathology spinobulbar muscular atrophy. ${ }^{8}$ Together, these studies suggested that in neuronal systems DNAJB2 mediates an increase in target protein degradation by the UPS, and that this activity participates in reducing the accumulation of cellular toxic protein aggregates. We therefore hypothesized a role for DNAJB2 in muscle degeneration rather than regeneration, and wished to study its possible participation in muscle degeneration pathologies, in particular those that involved protein aggregation and the UPS system. We have therefore enlarged our histological studies of human biopsies with a set of myofibrillar pathologies, characterized by the accumulation of protein aggregates and the formation of vacuoles inside the myofibers. This included sporadic inclusion body myositis, inclusion body myopathy with Paget's disease of bone and frontotemporal dementia, and genetically distinct myofibrillar myopathy, all of which are characterized by the accumulation of ubiquitin-positive protein aggregates.

The identified immunoreactivity of protein aggregates in all these pathologies is not specific to a single pathology and is not unique to DNAJB2, as we have shown also immunostaining for the $\alpha \mathrm{B}$-crystallin heat shock protein. However, we were particularly interested in sIBM pathology, since previous studies suggested that many other NMJ postsynaptic proteins are accumulated in intrafiber aggregates in sIBM. ${ }^{11}$ This includes amyloid $\beta$ precursor protein and its mRNA, prion protein and its mRNA, $\alpha$-synuclein and SMN (survival motor neuron) protein. ${ }^{11}$ Indeed, our extended study confirmed DNAJB2-positive aggregates in all seven studied SIBM biopsies. What could be the significance of DNAJB2 accumulation in muscle cytoplasmic aggregates in sIBM? DNAJB2 may play an active function at the muscle protein aggregates or perhaps only being passively associated with ubiquitinated aggregates. The hypothesis of simple passive attachment to ubiquitin positive aggregates is however rather unlikely, because this assumes also a passive diffusion, rather than an active shuttle, of DNAJB2 from the NMJ into the sarcoplasm, where it may meet the ubiquitinilated aggregate. The alternative "active role" hypothesis may implicate a possible failure of the proteasomal and/or autophagic protein degradation systems in the accumulation of DNAJB2 in sarcoplasmic aggregates. Indeed, malfunction of these systems has been proposed as one of the likely molecular mechanisms involved in the development of sIBM and other myofibrillar pathologies. ${ }^{12,13}$ Accordingly, the accumulation of ubiquitin-positive proteins in sarcoplasmic aggregates might reflect the reduced efficiency of their evacuation and degradation by these proteolytic systems. In such a scenario, since DNAJB2 may participate in targeting client protein(s) into the UPS degradation machinery, the slowing down of this pathway may result in the accumulation of DNAJB2 with its protein client in cytoplasmic aggregates. Interestingly, protein aggregates in myofibrillar myopathies were reported to be positive for p62, which is another ubiquitin interacting protein that plays a role in both the UPS and autophagic protein degradation. ${ }^{14}$

What role may DNAJB2 have in the NMJ of normal muscle? An important role has recently been proposed for the UPS in the homeostatic maintenance of synapses, including the one of the NMJ (Reviewed in 15,16). An involvement of chaperone proteins in this pathway is likely, in light of the recent discovery of HSP9O $\beta$ being expressed at the NMJ, where it has been suggested to regulate the clustering of the acetylcholine receptor via a direct interaction with rapsyn. ${ }^{17}$ This study further suggested that HSP9O $\beta$ regulates rapsyn degradation by the UPS. Interestingly, Schnaider et al, ${ }^{18}$ demonstrated a direct interaction of DNAJB2 with HSP9O in vitro, which in view of our data suggest their possible interaction in vivo. Being a heat shock associated protein and a mediator of protein degradation by the UPS, DNAJB2 joins HSP90 $\beta$ as a strong candidate for participating in the molecular mechanism of the homeostatic maintenance of the NMJ via the UPS proteolytic system.

Interestingly, in human only, we have identified in skeletal muscle higher expression of the $\mathrm{V} 1$ alternatively spliced isoform compared with the V2 isoform. The V1 DNAJB2 isoform doesn't have the CAAX consensus sequence for the geranylgeranylation post-translational modification that mediates attachment of DNAJB2 to internal membranes. Indeed, in transfected cells, the alternatively spliced V2 isoform has been shown to be localized to internal membranes and in particular to the cytoplasmic side of the endoplasmatic reticulum, while as the V1 isoform was localized to the cytoplasm and the nucleus (reference 5 and our own unpublished observations). Because unlike in human, the dominant muscle DNAJB2 isoform in the mouse is $\mathrm{V} 2$, it seems that localization of DNAJB2 to the NMJ is isoform-independent. Nevertheless, further detailed analysis of the muscle NMJ-specific DNAJB2 isoform in human and mouse will have to be addressed in future studies. At present therefore, the significance of the higher expression of $\mathrm{V} 1$ in human skeletal muscle, compared with V2 remains unexplained.

In summary, we have reported the expression of the co-chaperon DNAJB2 in mouse and human skeletal muscle; its localization at the neuromuscular junction in normal mature myofibers, and at the sarcoplasm and sarcolemma in regenerating fibers, and we have shown DNAJB2 accumulation in sarcoplasmic protein aggregates in diverse human protein aggregation myopathies.

\section{Acknowledgments}

We are grateful to the "Myobank" (Institute of Myology) for providing some of the muscle tissues, and to the BornBunge (Institute Biobank, Antwerp, Belgium) and its di- 
rector Prof Dr. Peter P. De Deyn for providing brain samples. We are very thankful to Isabelle Adamski, Christophe Georger, Bernard Gjata, Edith Peeters, Inge Bats, Evelyn Deleenheir, Maud Chapart, Maud Beuvin, Guy Brochier, Mai-Thao Bui, and Linda Manéré for their technical support.

\section{References}

1. Qiu XB, Shao YM, Miao S, Wang L: The diversity of the DnaJ/Hsp40 family, the crucial partners for Hsp70 chaperones. Cell Mol Life Sci 2006, 63:2560-2570

2. Cheetham ME, Brion JP, Anderton BH: Human homologues of the bacterial heat-shock protein DnaJ are preferentially expressed in neurons. Biochem J 1992, 284 (Pt 2):469-476

3. Mahoney DJ, Safdar A, Parise G, Melov S, Fu M, MacNeil L, Kaczor J, Payne ET, Tarnopolsky MA: Gene expression profiling in human skeletal muscle during recovery from eccentric exercise. Am J Physiol Regul Integr Comp Physiol 2008, 294:R1901-R1910

4. Banks GB, Chamberlain JS: The value of mammalian models for duchenne muscular dystrophy in developing therapeutic strategies. Curr Top Dev Biol 2008, 84:431-453

5. Chapple JP, Cheetham ME: The chaperone environment at the cytoplasmic face of the endoplasmic reticulum can modulate rhodopsin processing and inclusion formation. J Biol Chem 2003, 278:19087-19094

6. Chapple JP, van der Spuy J, Poopalasundaram S, Cheetham ME: Neuronal DnaJ proteins HSJ1a and HSJ1b: a role in linking the Hsp70 chaperone machine to the ubiquitin-proteasome system? Biochem Soc Trans 2004, 32:640-642

7. Westhoff B, Chapple JP, van der Spuy J, Hohfeld J, Cheetham ME: HSJ1 is a neuronal shuttling factor for the sorting of chaperone clients to the proteasome. Curr Biol 2005, 15:1058-1064

8. Howarth JL, Kelly S, Keasey MP, Glover CP, Lee YB, Mitrophanous K,
Chapple JP, Gallo JM, Cheetham ME, Uney JB: Hsp40 molecules that target to the ubiquitin-proteasome system decrease inclusion formation in models of polyglutamine disease. Mol Ther 2007, 15:1100-1105

9. Dubowitz V, CA S: A Practical Approach. London, Saunders Elsevier, 2007, pp. 3-39

10. Sanes JR, Lichtman JW: Induction, assembly, maturation and maintenance of a postsynaptic apparatus. Nat Rev Neurosci 2001 2:791-805

11. Askanas V, Engel WK, Alvarez RB: Fourteen newly recognized proteins at the human neuromuscular junctions-and their nonjunctional accumulation in inclusion-body myositis. Ann NY Acad Sci 1998, 841:28-56

12. Fratta P, Engel WK, McFerrin J, Davies KJ, Lin SW, Askanas V: Proteasome inhibition and aggresome formation in sporadic inclusion-body myositis and in amyloid-beta precursor protein-overexpressing cultured human muscle fibers. Am J Pathol 2005, 167:517-526

13. Lunemann JD, Schmidt J, Dalakas MC, Munz C: Macroautophagy as a pathomechanism in sporadic inclusion body myositis. Autophagy 2007, 3:384-386

14. Olive M, van Leeuwen FW, Janue A, Moreno D, Torrejon-Escribano B, Ferrer I: Expression of mutant ubiquitin $(\mathrm{UBB}+1)$ and p62 in myotilinopathies and desminopathies. Neuropathol Appl Neurobiol 2008, 34:76-87

15. Ding M, Shen K: The role of the ubiquitin proteasome system in synapse remodeling and neurodegenerative diseases. Bioessays 2008, 30:1075-1083

16. Haas KF, Broadie K: Roles of ubiquitination at the synapse. Biochim Biophys Acta 2008, 1779:495-506

17. Luo S, Zhang B, Dong XP, Tao Y, Ting A, Zhou Z, Meixiong J, Luo J, Chiu FC, Xiong WC, Mei L: HSP90 beta regulates rapsyn turnover and subsequent AChR cluster formation and maintenance. Neuron 2008, 60:97-110

18. Schnaider T, Soti C, Cheetham ME, Miyata Y, Yahara I, Csermely P Interaction of the human DnaJ homologue. HSJ1b with the $90 \mathrm{kDa}$ heat shock protein, Hsp90, Life Sci 2000, 67:1455-1465 